\title{
Nanoscale Structural and Chemical Properties of Ferroelectric Aluminum Scandium Nitride Thin Films
}

Pariasadat Musavigharavi ${ }^{1,2}$, Andrew C. Meng ${ }^{2}$, Dixiong Wang ${ }^{1}$, Jeffery Zheng ${ }^{2}$, Alexandre C. Foucher ${ }^{2}$, Roy H. Olsson III ${ }^{1}$, and Eric A. Stach*2,3

${ }^{1}$ Department of Electrical and Systems Engineering, University of Pennsylvania, Philadelphia, PA 19104 USA

2 Department of Materials Science and Engineering, University of Pennsylvania, Philadelphia, PA 19104 USA

${ }^{3}$ Laboratory for Research on the Structure of Matter, University of Pennsylvania, Philadelphia, PA 19104 USA

*Corresponding author: stach@seas.upenn.edu

\section{Supporting Information}
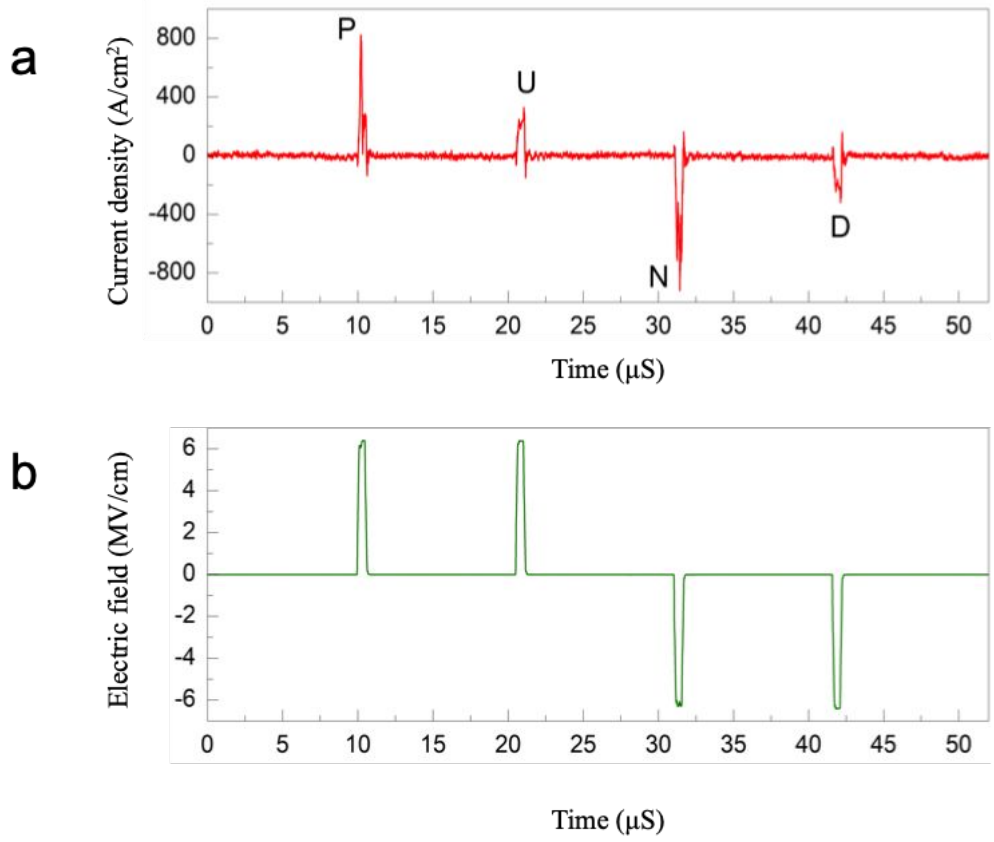

Figure S1 The PUND (a) currents and (b) electric field profile of the AlScN film. The P and $\mathrm{N}$ current peaks contain ferroelectric and non-ferroelectric contributions, while the $\mathrm{U}$ and $\mathrm{D}$ sequences only contain the non-ferroelectric behaviors. 

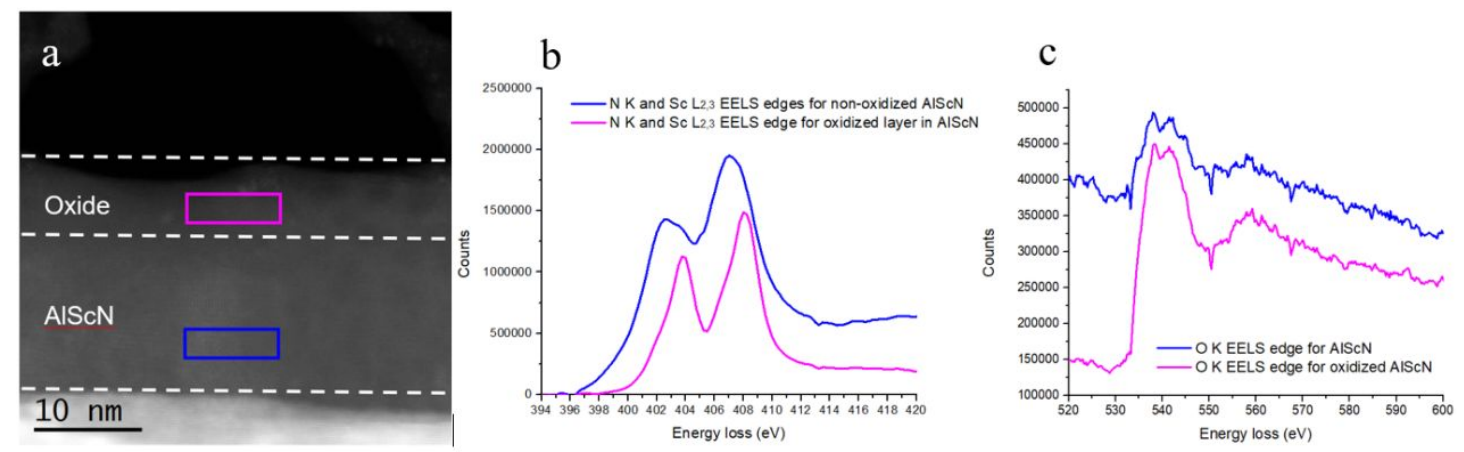

Figure S2 EELS investigation of nitrogen in AlScN film. (a) Dark-field STEM image of the AlScN film, with two regions that were analyzed with EELS. (b) $\mathrm{N} \mathrm{K}$ edge in the EELS spectrum for the oxide layer (pink) and non-oxidized $\mathrm{AlScN}$ (blue). The peaks are shifted by $1.5 \mathrm{eV}$ between the oxide and the rest of the thin film. (c) O K EELS edge for the two regions: No shift or change in the shape of the O K EELS edge is observed.
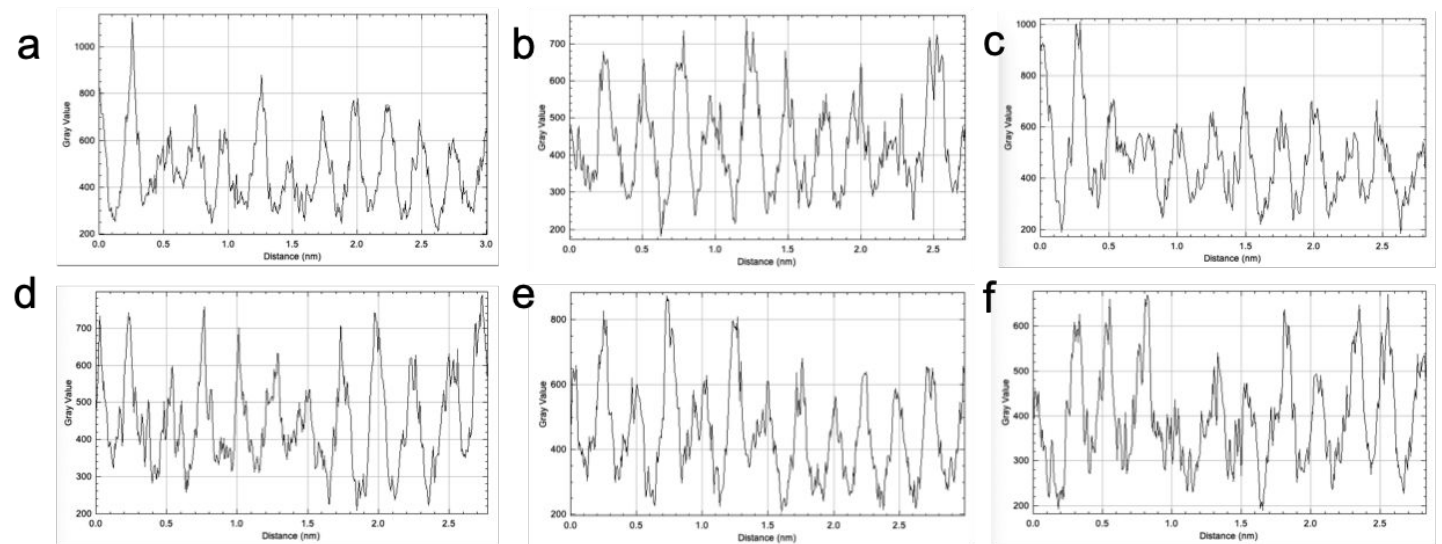

Figure S3 (a-f) The representative intensity-distance line profiles across the row of atomic planes on top/left of the film (Trace 1), used to calculate the average $d$-spacing (using Digital Micrograph). The average interplanar distance $\left(d_{\text {spacing }}\right)$ is $2.50 \pm 0.02 \AA$, corresponding to the (0002) planes of the wurtzite crystal structure in the AlScN film. This approach is used to calculate the average and the standard deviation of the interplanar spacing. 